\title{
Examen psicométrico del IQ Test como herramienta de discriminación de individuos normales y talentosos en la población escolar chilena*
}

\author{
Psychometric Examination of the IQ Test as a Tool of Discrimination \\ of Normal and Talented Individuals in the Chilean School Population
}

Enviado: 12 de diciembre de 2014 | Revisado: 25 de junio de 2015 | Aceptado: 25 de junio de 2015

\author{
Gamal Cerda Etchepare** \\ CARLOS PÉrez WILSON *** \\ Universidad de Concepción, Chile \\ ROBERTO MELIPILlÁn ARANEDA**** \\ Universidad del Desarrollo, Chile \\ ROSARIO ORTEGA-RUIZ ***** \\ Universidad de Córdoba, España
}

doi:10.11144/Javeriana.upsy14-3.epiq

Para citar este artículo: Cerda, G., Pérez, C., Melipillán, R., \& Ortega-Ruiz, R. (2015). Examen psicométrico del IQ Test como herramienta de discriminación de individuos normales y talentosos en la población escolar chilena. Universitas Psychologica, 14(3), 899-912. http://dx.doi.org/10.11144/ Javeriana.upsy14-3.epiq

"Trabajo apoyado por el Proyecto Basal FB0003 del Programa de Investigación Asociativa de Conicyt, y por el proyecto Fondecyt regular n⿳ำ 1130519 del gobierno de Chile

** Profesor asociado, Facultad de Educación, Departamento de Metodología de la Investigación e Informática Educacional. Investigador asociado, Centro de Investigación Avanzada en Educación, CIAE.Correo electrónico: gamal.cerda@udec.cl

**** Profesor asociado, Facultad de Ciencias Físicas y Matemáticas, Departamento de Ingeniería Matemática. Investigador asociado, Centro de Investigación Avanzada en Educación, CIAE. Correo electrónico: carlos.perez@udec.cl

****** Docente-Investigador, Facultad de Psicología, Universidad del Desarrollo. Correo electrónico: robmeli@umich.edu

******* Catedrática, Departamento de Psicología, Universidad de Cordoba. Directora del Programa de Doctorado "Psicología Aplicada" y del programa de "Máster Oficial en Intervención e Investigación Psicológica en Justicia, Salud y Bienestar Social". Correo electrónico: ed1orrur@uco.es

\section{RES UMEN}

Se presentan los resultados del proceso de adaptación del IQ Test del grupo Mensa Dinamarca, para aplicarlo en Chile y así discriminar grupos de capacidad intelectual diferenciada. Se aplicó el instrumento a dos muestras de estudiantes secundarios chilenos: 2994 (grupo normal) y 394 (grupo aventajado). El instrumento presenta adecuados índices de fiabilidad, confirmando la validez del constructo mediante análisis factoriales y de Rasch. La validez concurrente se constató al comparar los desempeños con otro test de inteligencia previamente estandarizado en la población escolar chilena. El instrumento logra diferenciar individuos en los dos grupos (normal y aventajado) y sus puntuaciones se correlacionan positivamente con el rendimiento en matemáticas. Se discuten las principales implicaciones del estudio para el sistema escolar chileno.

Palabras clave

inteligencia lógica; matemáticas; rendimiento escolar

\section{A B S T R A C T}

This article presents results from the process of adaption of the Mensa Denmark IQ Test in Chile. The test was adapted to discriminate between groups of Chilean students with different intellectual capacities. The instrument was applied to two samples of Chilean secondary students: 2994 (normal group) and 394 (advanced group). The instrument shows adequate levels of confidence, and the validity of the instrument was confirmed by means of factor analysis and Rasch analysis. Concurrent validity was established when comparing the performance of the Chilean school population in other types of previously standardized intelligence tests. This instrument is able to differentiate individuals in both groups (normal and advanced) and their scores correlate positively with their performance in mathematics. This article discusses the main implications of this study for the Chilean school system. Keywords

logical intelligence; mathematics; school performance 


\section{Introducción}

Generalmente en el ámbito educativo los test de inteligencia complementan la evaluación de programas educativos, también se convierten en uno de los factores a considerar a la hora de explicar el rendimiento académico (Deary, Strand, Smith $\&$ Fernandes, 2007). A partir de ello, la administración del test a la población escolar permite el diagnóstico y pronóstico de las potencialidades o eventuales dificultades de los estudiantes en su desempeño académico y aprendizaje escolar (Watkins, Lei \& Canivez, 2007).

Actualmente parece existir un amplio consenso respecto al carácter predictivo del nivel de inteligencia general de un individuo con su desempeño profesional, su capacidad para resolver problemas y el éxito o fracaso escolar (Almeida, Guisande, Primi \& Lemos, 2008; Blackwell, Trzesniewski \& Sorich, 2007; Deary et al, 2007; Furnham, Monsen $\&$ Ahmetoglu, 2009; Kotz, Watkins \& McDermott, 2010; Steinmayr, Ziegle \& Träuble, 2010). También existe evidencia de una asociación positiva con la memoria y tareas de naturaleza viso-espacial (Ferreira, Almeida, Prieto \& Guisande, 2012; Johnson $\&$ Bouchard, 2005). Sin embargo, factores tales como autoestima, salud, morbilidad, situación económica e incluso problemas con la justicia, matizan esa relación, tal y como lo han puesto de manifiesto diversas investigaciones (Arden, Gottfredson \& Miller, 2009; Deary, Taylor, Hart, Wilson, Smith \& Blane, 2005; Gottfredson \& Deary, 2004; Kornilova, Kornilov \& Chumakova, 2009; Rindermann, 2008, por nombrar algunas).

El potencial cognitivo muestra una trayectoria consistente y estable a medida que se recorre el itinerario escolar, mostrando diferencias significativas entre hombres y mujeres (Roselli, Ardila, Matute \& Inozemtseva, 2009; Ferrándiz, Bermejo, Sainz, Ferrando \& Prieto, 2008; Kaufman, Kaufman, Liu \& Johnson, 2009; Klein, Adi-Jaha \& HakakBenizri, 2010). Esto último es relevante, pues aun cuando se observan diferencias en áreas específicas de aprovechamiento académico como las matemáticas, estas parecen obedecer más a patrones culturales y de equidad de género que a diferencias de capacidades básicas (Else-Quest, Hyde \& Linn, 2010). La inteligencia general es un predictor importante del rendimiento escolar en diversas materias académicas y especialmente en matemáticas (Deary et al., 2007; Furnham et al. 2009; Kotz et al., 2010; Lynn \& Mikk, 2009; Spinath, Freudenthaler \& Neubauer, 2010).

La evidencia de la inteligencia como factor general, se acentúa para la denominada inteligencia fluida o no verbal, que se erige como uno de los mejores predictores simples del desempeño escolar (Almeida et al, 2008). La inteligencia fluida alude a la capacidad para adaptarse y afrontar situaciones nuevas de forma flexible sin que el aprendizaje previo constituya una fuente de ayuda determinante para su manifestación (Almeida, 2008; Kvist \& Gustafson, 2008). La inteligencia fluida permite razonar con contenidos abstractos, establecer relaciones o extraer diferencias y razonar lógicamente, lo que sugiere su importancia en el desarrollo de las habilidades matemáticas (Dodonova \& Dodonov, 2012; Gullick, Sprute \& Temple, 2011). Este tipo de inteligencia parece alcanzar su máximo desarrollo en la adolescencia, de ahí la intención de contribuir con un nuevo instrumento para evaluar la población escolar de secundaria en Chile.

Por otra parte, en los últimos años en Chile se ha encontrado evidencia de la relación positiva de la inteligencia lógica con las estrategias de aprendizaje de tipo elaboradas y profundas, constatando diferencias significativas respecto a la edad, género y extracción social en estudiantes de diversos niveles educativos (Cerda, Ortega, Pérez, Flores \& Melipillán, 2011), lo que resulta coincidente con las diferencias observadas en el rendimiento o aprovechamiento escolar -en matemáticas y diversas áreas a nivel nacional e internacional- que han podido ser observadas en aplicaciones de instrumentos en diversos niveles y materias al estudiantado chileno e internacional (Crosnoe \& Huston, 2007; MINEDUC, 2007a, 2007b, 2010a; 2010b).

En lo referido a los alumnos denominados 'talentosos' o de rendimiento académico superior, se ha reportado evidencia consistente respecto a la existencia de diferencias significativas en cuanto a las habilidades lógicas y matemáticas que poseen 
(Cerda, Pérez \& Melipillán, 2010) frente a grupos no seleccionados. Esto puede ser consecuencia de que aquellos alumnos con un elevado rendimiento en matemáticas poseen habilidades cognitivas de alto nivel, como lo son la planificación, revisión, control, selección y evaluación de las propias actividades intelectuales (Onrubia, Rochera \& Barberá, 2003). De hecho, otro estudio con alumnos talentosos en Chile señala que según sus profesores, estos alumnos presentan características académicas y socio-afectivas bastante similares al resto, con la excepción del dominio específico de lógica y matemáticas (Flanagan \& Arancibia, 2005). En el mismo sentido, en aquellos educadores que tienden a identificar el coeficiente intelectual como base para la determinación del talento de sus estudiantes, generalmente destacan las habilidades analíticas como parte fundamental de la estructura de la inteligencia por encima de características personales (García-Cepero \& McCoach, 2009).

En Chile se ha avanzado de manera notoria en acceso y cobertura en educación, pero con pocos logros en calidad y equidad de los aprendizajes, siendo esta una situación bastante crítica en los sectores vulnerables (Cox, 2007). Un diagnóstico de las capacidades de inteligencia fluida de los alumnos podría permitir estructurar mejor la planificación de aula o separar grupos para un mejor aprovechamiento escolar, especialmente en matemáticas. En el ámbito de esta disciplina escolar, los estudiantes requieren reconocer patrones o dar sentido a un material desorganizado o confuso y encontrar o identificar reglas subyacentes a una serie de estímulos figurativos como los que examina el instrumento examinado en la presente investigación.

El IQTest del Grupo Mensa Dinamarca, diseñado por Anders Ditlev Jensen, es un instrumento de libre acceso disponible en www.iqtest.dk. Es un test figurativo, de series incompletas y de selección múltiple que considera matrices lacunarias de elección múltiple, todos ellos aspectos similares a aquellos del Test de matrices progresivas de Raven (Raven, Raven \& Court, 1993). Además, en su resolución no se involucran procesos de lectura comprensiva, manejo de información o conocimiento previo.
Por lo anterior, se desprende el potencial que el IQ Test puede tener para evaluar la capacidad de razonamiento lógico-matemático en la población escolar chilena. Sin embargo, para que este instrumento sirva a tal propósito, debe reunir los atributos y características psicométricas exigibles a un instrumento con tal condición, que es el objetivo de investigación de este trabajo. Es por esta razón que la investigación intenta responder a las siguientes preguntas:

- iTendrá el IQ Test las características psicométricas adecuadas para ser considerado un instrumento válido y confiable en la población escolar chilena?

- ¿Permitirá este instrumento diferenciar entre aquellos estudiantes con capacidades académicas superiores?

- iSe relacionarán las puntuaciones de este instrumento con el rendimiento académico general y en particular, con el rendimiento en matemáticas?

\section{Método}

De acuerdo a los objetivos del estudio, la investigación se enfoca en un paradigma cuantitativo con un diseño descriptivo correlacional, dado que se intenta describir las características y relaciones que presenta el fenómeno en estudio.

\section{Participantes}

Se realizó una aplicación piloto en una primera muestra de 200 estudiantes con el objeto de ver la adecuada comprensión de las instrucciones y si los objetos dibujados eran reconocidos adecuadamente por los estudiantes, además de otros factores que pudieran distorsionar las respuestas. Sobre esta misma muestra se realizaron análisis de carácter preliminar de los ítems y de la fiabilidad del instrumento. El análisis de la aplicación piloto indicó la existencia de seis ítems cuyas cualidades discriminativas y de carga no resultaban ajustadas a las normas para este efecto. Sin la inclusión de ellos, el instrumento en general presentó índices de fiabilidad y de validez muy adecuados. 
Luego de la etapa piloto se procedió a seleccionar una muestra definitiva de proporción estratificada por nivel educativo. En Chile hay evidencia que indica que el grupo de extracción social (alta, media y baja) es homologable al tipo de dependencia administrativa de los establecimientos en el sistema educativo (particular, subvencionada y municipalizada, respectivamente) García-Huidobro y Bellei (2003). La muestra definitiva se definió en base a criterios de nivel educativo, género y edad de los alumnos a lo largo del país, de acuerdo a lo que muestra la Tabla 1. Adicionalmente se decidió considerar en la aplicación del instrumento un grupo adicional (separado) de estudiantes destacados en matemáticas, a los cuales también se les aplicó el test. Este grupo de estudiantes considerados destacados en matemáticas correspondió al grupo de estudiantes que participan en la competencia "Campeonato Regional Escolar de Matemáticas, CEMAT".

La muestra total tiene una edad promedio de 14.13 años y una desviación típica de 1.687. Por su parte, la muestra de los estudiantes denominados como talentosos alcanzó un total de 394 estudiantes distribuidos de la siguiente manera: 142 estudiantes provenientes de establecimientos de nivel socioeconómico alto, 201 estudiantes de establecimientos de nivel socioeconómico medio y 51 estudiantes de establecimientos de nivel socioeconómico bajo, con una edad promedio de 14.22 años y una desviación típica de 1.641. Del total de alumnos talentosos, 226 son hombres y 168 mujeres.

\section{Procedimiento}

La investigación se realizó en establecimientos educacionales de la región del Bío Bío, Chile. Al considerar la aplicación de instrumentos y recopilación de antecedentes socio académicos, se siguió la normativa APA en lo referido a Principios éticos y códigos de conducta, lo que implicó entre otras cosas, contactar a los establecimientos educacionales y planificar las acciones a realizar al interior de los establecimientos. Para ello se tuvo que contar con el consentimiento informado de los estudiantes, padres o tutores y las autoridades de los establecimientos educativos, con respecto a la confidencialidad de los resultados y su uso restringido a los fines de la presente investigación.

Una vez aplicados los instrumentos, se tabularon las respuestas en planillas de registro, en las cuales se incorporaron aquellos antecedentes individuales relacionados con el rendimiento académico extraídos directamente de las actas de rendimiento escolar.

\section{Instrumentos}

IQ Test: Cuenta con 39 ítems y un tiempo de administración de 40 minutos. Cada ejercicio presenta la estructura que indica la Figura 1 a la izquierda: al centro se presenta una estructura con 9 recuadros, en donde el recuadro final de la serie está vacío y debe ser completado con alguna de las 8 alternativas propuestas en la parte baja del mismo. Para el proceso de aplicación colectiva, se generó una

TABLA 1.

Distribución de la muestra final de estudiantes en función de la extracción social de los establecimientos a los cuales asisten y su edad (años) para el IQ Test

\begin{tabular}{llllllll}
\hline \multirow{2}{*}{ Clase social } & EDAD & & & & & & \multirow{2}{*}{ Total } \\
\cline { 2 - 7 } & 12 & 13 & 14 & 15 & 16 & 17 & 151 \\
\hline Alta & 15 & 44 & 29 & 30 & 17 & 16 & 1419 \\
Media & 134 & 245 & 306 & 347 & 256 & 131 & 1424 \\
Baja & 89 & 158 & 305 & 365 & 304 & 203 & 2994 \\
\hline Total & 238 & 447 & 640 & 742 & 577 & 350 & \\
\hline
\end{tabular}

Fuente: elaboración propia 

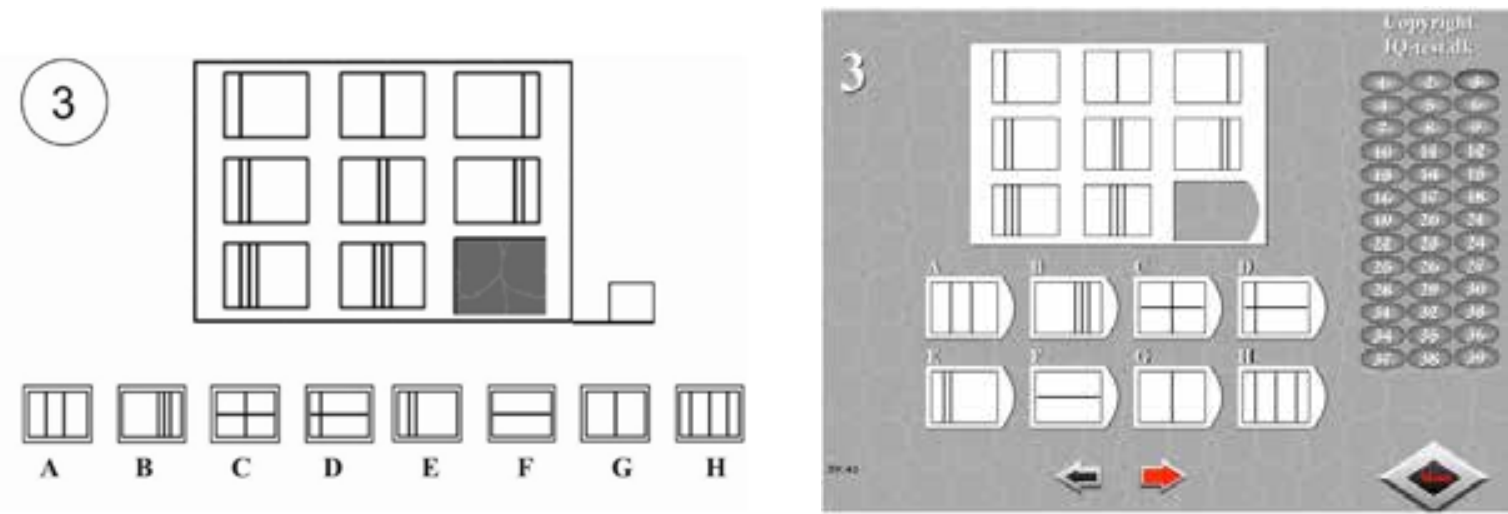

Figura 1: Versiones web (derecha) e impresa (izquierda) de uno de los ítems del IQ Test considerados para el trabajo de investigación

Fuente: elaboración propia

versión impresa en la que se enumeró cada ejercicio y las alternativas propuestas se dispusieron en una sola fila abajo de la estructura para poder presentar varios ejercicios en una misma hoja. Se presentan ambos formatos, virtual e impreso respectivamente, en la Figura 1.

Para facilitar la comprensión de la tarea exigida por el test, se confeccionaron tres ítems adicionales como ejemplos que permiten comprender la forma en que debe ser resuelto y que no forman parte del examen final. Este test permite medir aspectos de la inteligencia fluida, que evidencia relación con la capacidad de dar sentido a un material desorganizado o confuso al reconocer patrones o reglas subyacentes a una serie de estímulos figurativos.

Test de inteligencia lógica superior (TILS): Consta de 50 ítems y un tiempo de administración de 30 minutos. En su protocolo de aplicación considera cinco ejemplos adicionales que permiten entender la forma en que se debe responder. Sus ítems son de tipo figurativo, incluyendo formas geométricas abstractas como puntos, líneas rectas o curvas, polígonos, etc. Cada ítem o reactivo presenta la misma estructura tipo, debidamente numerada. En el sector izquierdo de la hoja existen cuatro figuras de una serie unidas por alguna regla o patrón. A esa serie hay que agregarle una quinta figura que continúe dicha secuencia, para lo cual la persona debe elegir la alternativa correcta entre cinco posibilidades que se presentan. El alfa de Cronbach es 0.94. La Figura 2 muestra dos ítems que forman parte de los ejemplos de inducción del instrumento.

Este test mide la inteligencia lógica de carácter inductiva, entendida como la capacidad que tienen las personas para vislumbrar soluciones y resolver problemas, estructurar elementos para realizar deducciones y fundamentarlas con argumentos
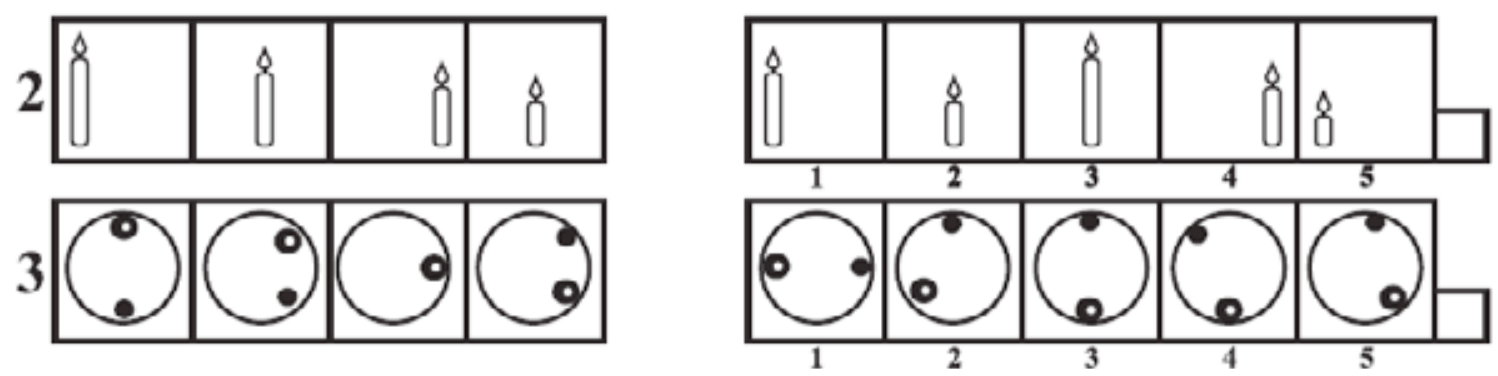

Figura 2: Ejercicios tipo del TILS

Fuente: elaboración propia 
sólidos. Involucra la capacidad para reconocer en una serie de elementos, la regla general que los rige o que subyace a los mismos.

Con respecto a las variables asociadas al rendimiento académico, se han considerado dos: el rendimiento general entendido como el promedio de calificaciones que obtienen los estudiantes de los diversos cursos de educación secundaria en las asignaturas que cursan en un año lectivo, y el rendimiento en matemáticas, entendido como el promedio de las calificaciones en la asignatura o curso de matemáticas en esa disciplina escolar en el mismo año lectivo.

\section{Análisis de datos}

Como se mencionó anteriormente, el principal objetivo del estudio es establecer si el IQ Test reúne adecuados niveles de validez y fiabilidad en población escolar chilena. En el caso de la evaluación de la validez del constructo se llevó a cabo un análisis de Rasch para ítems dicotómicos (Embretson \& Reise, 2000). Una ventaja de este tipo de análisis en comparación a aquellos derivados de la teoría clásica de los test, es que esta última trata todos los ítems como equivalentes, ignorando la interacción de los niveles del rasgo latente implicados en los ítems con el nivel del rasgo en los individuos, que pueden producir patrones de respuesta particulares (Muñiz, 2010).

El modelo de Rasch asume que la probabilidad de contestar favorablemente un ítem es una función logística de la diferencia entre el nivel del rasgo latente exhibido por un individuo y el nivel de dificultad del ítem, esto es:

$$
p_{i}(\theta)=\frac{e^{\left(\theta-b_{i}\right)}}{1+e^{\left(\theta-b_{i}\right)}}
$$

En la expresión anterior, $p_{i}(\theta)$ es la probabilidad de que un individuo con un nivel $\theta$ del rasgo latente responda afirmativamente al ítem $i$, mientras que $b_{i}$ es el parámetro de dificultad de ese ítem.

Para una adecuada interpretación de los resultados obtenidos en un modelo de Rasch, se requiere que los datos satisfagan los supuestos de unidimensionalidad e independencia local. El supuesto de unidimensionalidad se refiere a que los ítems analizados midan un único rasgo latente. Para evaluar este supuesto se sometieron los ítems de la escala a un análisis factorial exploratorio (AFE). Por su parte, el supuesto de independencia local implica que cuando se controlan los niveles del rasgo latente medido, las respuestas a los distintos ítems resultan independientes entre sí. Así las cosas, se está en presencia de dependencia local si las respuestas a distintos ítems muestran una covariación que se explica por factores distintos (ej. contenido o fraseo similar de los ítems) a la relación común que dichos ítems mantienen con el rasgo latente medido. Para determinar si los ítems de la escala satisfacían este supuesto, se llevó a cabo un análisis de componentes principales sobre los residuos del modelo. Como criterios para determinar la existencia de dependencia local entre los ítems se consideró: a) la presencia de componentes principales que explicaran más de dos unidades de varianza (Linacre, 2010) y b) que dichos componentes principales mostraran un número suficiente de ítems con cargas que excedieran el criterio |0.30|.

Finalmente se analizó el ajuste de cada uno de los ítems al modelo de Rasch empleando los estadísticos Infit y Outfit. Estos estadísticos permiten detectar anomalías en el ajuste de los datos a las características del modelo, tales como la presencia de inconsistencias en los patrones de respuestas, o bien frente a la presencia de ítems con distintos niveles de discriminación. Como criterio para interpretar la existencia de un buen ajuste se propone que los valores Infit y Outfit de cada ítem deberían localizarse dentro del intervalo 0.5 a 1.5 (Linacre, 2010).

Junto a lo anterior, también se procedió a evaluar la validez concurrente mediante el examen de correlaciones producto-momento de Pearson entre las variables-criterio rendimiento académico general, rendimiento en matemáticas e inteligencia lógica. En cuanto a los análisis de confiabilidad, se empleó el coeficiente alfa de Cronbach para evaluar la consistencia interna de los ítems.

Adicionalmente, se realizaron análisis de varianza simple y de diferencia de medias para determinar posibles diferencias en función de edad, 
género y grupo de extracción social, además de la prueba de Levene para determinar la homogeneidad de las varianzas y el cumplimiento del supuesto de normalidad. Para realizar estos análisis se utilizaron los programas SPSS 19, MPLUS 6 y WINSTEPS 3.70.0.

\section{Resultados}

El IQ Test presenta un adecuado nivel de fiabilidad $\left(r_{\alpha}=0.81\right)$ y el análisis de sus ítems demuestra que exhiben en general, grados de dificultad normales y niveles de discriminación y homogeneidad adecuados y moderados.

\section{Evaluación de la dimensionalidad}

Para verificar el supuesto de unidimensonalidad se sometieron 33 de los ítems del IQ Test que permanecieron después de la aplicación piloto, a un análisis factorial exploratorio (AFE) para examinar si se relacionaban con un único factor. La calidad del ajuste se llevó a cabo empleando e interpretando los estadísticos CFI, TLI y RMSEA (Albright \& Park, 2009). Las soluciones factoriales para uno y dos factores se presentan en la Tabla 2.

Como se observa en la Tabla 2, los dos modelos factoriales analizados mostraron un adecuado ajuste a los datos, puesto que presentaron un CFI y TLI del orden de 0.95 , así como un coeficiente RMSEA menor a 0.05 , considerados todos ellos como valores apropiados para un modelo factorial (Albright \& Park, 2009). Al complementar el análisis de los resultados anteriores con el examen de las matrices de configuración de las dos soluciones factoriales se concluyó que el modelo de un factor resultaba ser el más apropiado para efectos de interpretación sobre la base de la matriz de coeficientes de configuración para esta solución factorial, como lo muestra la Tabla 3.

TABLA 2.

Calidad del ajuste a los datos obtenidos por los modelos de uno y dos factores para el IQ Test

\begin{tabular}{cccccc}
\hline Modelo & $\chi 2(\mathrm{gl})$ & CFI & TLI & RMSEA & SRMR \\
\hline 1 Factor & $2091.43 * * *(495)$ & 0.94 & 0.94 & 0.03 & 0.06 \\
2 Factores & $1037.40 * * *(463)$ & 0.98 & 0.98 & 0.02 & 0.04 \\
\hline
\end{tabular}

$* * * \mathrm{p}<0.001$

Fuente: elaboración propia

\section{TABLA 3.}

Matriz de configuración para la solución de un factor del IQ Test

\begin{tabular}{cccccccc}
\hline Ítem & Carga & Ítem & Carga & Ítem & Carga & Ítem & Carga \\
\hline 1 & 0.77 & 10 & 0.38 & 19 & 0.52 & 28 & 0.52 \\
\hline 2 & 0.71 & 11 & 0.24 & 20 & 0.22 & 29 & 0.27 \\
\hline 3 & 0.81 & 12 & 0.56 & 21 & 0.31 & 30 & 0.36 \\
\hline 4 & 0.78 & 13 & 0.60 & 22 & 0.46 & 31 & 0.20 \\
\hline 5 & 1.00 & 14 & 0.29 & 23 & 0.54 & 32 & 0.37 \\
\hline 6 & 0.86 & 15 & 0.69 & 24 & 0.54 & 33 & 0.33 \\
\hline 7 & 0.72 & 16 & 0.54 & 25 & 0.37 & & \\
\hline 8 & 0.74 & 17 & 0.63 & 26 & 0.31 & & \\
\hline 9 & 0.66 & 18 & 0.60 & 27 & 0.22 & & \\
\hline
\end{tabular}

Fuente: elaboración propia 
Basados en este resultado podemos concluir que el IQ Test tiene una estructura adecuada para ser sometido a un análisis de Rasch.

\section{Dependencia local}

Una vez ajustado el modelo de Rasch, se procedió a evaluar si las respuestas a los distintos ítems mostraban alguna asociación no explicada por el modelo. Para ello se llevó a cabo un análisis de componentes principales de los residuos. En cuanto a la varianza residual explicada por los componentes extraídos, se observó que la máxima varianza alcanzó un valor de 1.7 unidades, el cual resulta inferior al criterio de 2 unidades señalado como indicador de la presencia de un componente residual (Albright \& Park, 2009).

\section{Ajuste de los items al modelo de Rasch}

$\mathrm{Al}$ analizar los valores obtenidos por los ítems del instrumento en el estadístico Outfit, se observó que 28 de los 33 ítems de la etapa piloto (85\%) presentaron un valor igual o inferior a 1.5 , lo cual permite concluir que dichos ítems contribuyen a definir un único rasgo latente. Los ítems que exhibieron valores superiores a este valor fueron: 20, 31, 27, 21 y 30. Dado el inadecuado ajuste evidenciado por estos ítems, se procedió a su eliminación, luego de lo cual se volvió a repetir el análisis de Rasch con los 28 ítems seleccionados. Los resultados de esta depuración se sometieron a un nuevo análisis cuyos resultados se presentan en la Tabla 4.

La Figura 2 permite observar gráficamente la distribución de las medidas de habilidad de los participantes y la dificultad de los 28 ítems resultantes del IQ Test en el mismo continuo lineal generado por el modelo de Rasch. A la izquierda de la línea vertical se presentan los resultados de los participantes. En la parte superior se localizan los participantes con mayores niveles de habilidad. En la parte inferior se localizan los participantes con menor nivel de habilidad. A la derecha de la línea vertical se presentan los resultados de los ítems. Los ítems con mayor nivel de dificultad se localizan en la parte superior. Los ítems con menor nivel de dificultad se localizan en la parte inferior. Al comparar el promedio en la escala logits del modelo de Rasch obtenido por la muestra de participantes $(\mathrm{M}=1.05, \mathrm{EE}=0.53)$ y por los ítems del test $(M=0.00, E E=0.05)$, se observa que la mayoría de los participantes están localizados sobre el valor 0.00 en la escala logits.

TABLA 4

Análisis de Rasch para los ítems de la versión final del IQ Test, ordenados según su medida

\begin{tabular}{cccccccccc}
\hline Ítem & Medida & EE & Infit & Outfit & Ítem & Medida & EE & Infit & Outfit \\
\hline 26 & 2.03 & 0.04 & 1.09 & 1.27 & 23 & 0.55 & 0.04 & 0.96 & 0.99 \\
33 & 2.03 & 0.04 & 1.05 & 1.33 & 16 & 0.05 & 0.04 & 1.00 & 1.07 \\
29 & 1.84 & 0.04 & 1.14 & 1.43 & 15 & -0.02 & 0.04 & 0.87 & 0.79 \\
22 & 1.77 & 0.04 & 0.98 & 1.21 & 13 & -0.09 & 0.04 & 0.95 & 0.94 \\
14 & 1.75 & 0.04 & 1.12 & 1.41 & 17 & -0.36 & 0.04 & 0.93 & 0.89 \\
10 & 1.57 & 0.04 & 1.06 & 1.24 & 9 & -0.94 & 0.05 & 0.94 & 0.98 \\
25 & 1.36 & 0.04 & 1.07 & 1.33 & 7 & -1.23 & 0.06 & 0.91 & 0.94 \\
32 & 1.19 & 0.04 & 1.07 & 1.22 & 4 & -1.85 & 0.07 & 0.88 & 0.78 \\
19 & 1.10 & 0.04 & 0.96 & 1.09 & 6 & -2.07 & 0.07 & 0.80 & 0.58 \\
12 & 1.00 & 0.04 & 0.94 & 0.95 & 8 & -2.10 & 0.07 & 0.88 & 1.11 \\
18 & 0.97 & 0.04 & 0.89 & 1.04 & 2 & -2.21 & 0.08 & 0.97 & 1.10 \\
28 & 0.85 & 0.04 & 0.96 & 1.04 & 3 & -2.24 & 0.08 & 0.85 & 0.90 \\
11 & 0.81 & 0.04 & 1.22 & 1.35 & 1 & -3.20 & 0.11 & 0.90 & 0.80 \\
24 & 0.66 & 0.04 & 0.96 & 1.01 & 5 & -3.22 & 0.11 & 0.71 & 0.25 \\
\hline
\end{tabular}

Fuente: elaboración propia 


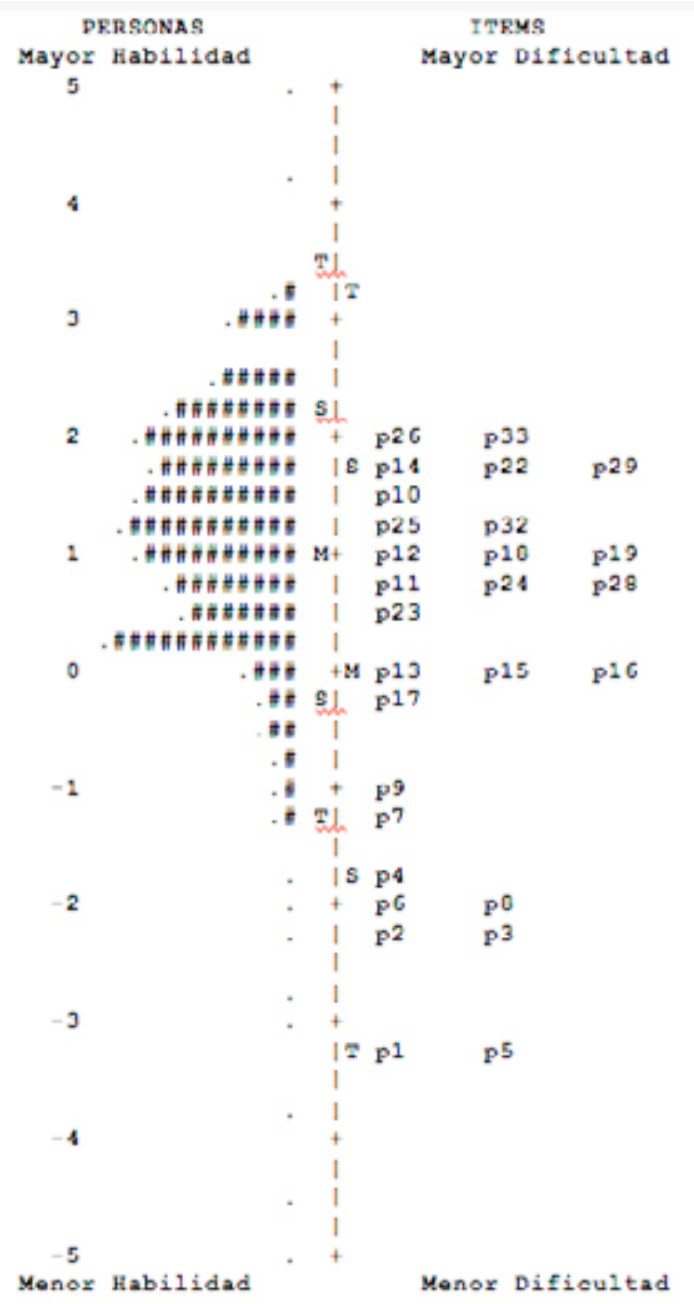

Figura 3. Distribución de las medidas de habilidad de los participantes y la dificultad de los 28 items del IQ Test en el mismo continuo lineal generado por el modelo de Rasch

Fuente: elaboración propia

\section{Validez de criterio}

La validez de criterio se analizó mediante el estudio de las correlaciones producto-momento de Pearson entre las puntuaciones del test y las variables de criterio: promedios de notas general y promedio de notas en matemática, también respecto al puntaje alcanzado por los mismos estudiantes en el TILS que se aplicó previamente a la misma población escolar y que está validado y estandarizado en Chile (Cerda et al., 2011). Como se observa en la Tabla 5, las correlaciones permiten avalar la validez de criterio (rendimiento académico) y también la validez concurrente (inteligencia lógica) del IQ Test. El análisis previo de los gráficos de dispersión permitió constatar la inexistencia de relaciones no-lineales entre las variables, así como la ausencia de valores atípicos influyentes.

Como se observa en la Tabla 5, el IQ Test muestra correlaciones significativas en la dirección esperada con cada una de las variables de criterio. Se observa que el test exhibe relaciones directas y significativas con el promedio de calificaciones general de los estudiantes $r(2770)=0.388, p<$ 0.001 y con el promedio de calificaciones en matemáticas $r(2924)=0.425, p<0.001$. Al analizar las correlaciones entre el IQ Test y la escala TILS, la correlación observada corresponde a $r(2934)=$ 
TABLA 5.

Matriz de correlaciones producto-momento de Pearson para el IQ Test, TILS y promedios de notas

\begin{tabular}{lcccc}
\hline \multicolumn{1}{c}{ Variables } & 1 & 2 & 3 & 4 \\
\hline 1: IQ Test & - & $0.616^{* * *}$ & $0.388^{* * * *}$ & $0.425^{* * *}$ \\
2: TILS & & - & $0.379^{* * *}$ & $0.439^{* * * *}$ \\
3: Promedio general & & & - & $0.728^{* * *}$ \\
4: Promedio matemáticas & & & & - \\
\hline$* * * \mathrm{p}<0.001$ & & & & \\
Fuente: elaboración propia & & &
\end{tabular}

$0.616, p<0.001$, que resulta altamente significativa. En cuanto al tamaño del efecto asociado a las relaciones identificadas, se observa que la relación más intensa se produce al correlacionar las puntuaciones del IQ Test y TILS $(r \geq|0.50|)$, mientras que las restantes correlaciones analizadas exhiben relaciones de moderada intensidad $(r \approx|0.30|)$.

Todo lo anterior permite concluir que el IQ Test posee adecuados atributos de validez de criterio para utilizarse al analizar los niveles de inteligencia inductiva de los estudiantes de la población escolar chilena, esto se presenta en las secciones siguientes.

\section{Análisis de los niveles de inteligencia inductiva de los estudiantes}

$\mathrm{Al}$ analizar los puntajes obtenidos en el IQ Test en función de la edad se constata la existencia de diferencias significativas en las medias de las puntuaciones en función de la edad de los alumnos que lo rinden $[\mathrm{F}(5.2990)=21.057, \mathrm{p}<0.001]$. Se observa un incremento consistente a medida que los alumnos maduran cognitivamente o tienen más edad. Con respecto a las puntuaciones alcanzadas por hombres y mujeres se observa que no existen diferencias significativas $(\mathrm{t}(3132)=-2.231$, n.s.) aun cuando la puntuación media alcanzada por las mujeres es levemente superior $(\mathrm{M}=19.01, \mathrm{DT}=5.267)$ y $(M=18.58, D T=5.306)$ respectivamente.

Del mismo modo, los resultados arrojaron diferencias significativas al comparar las puntuaciones alcanzadas por los estudiantes en función del grupo de extracción social $[\mathrm{F}(2.3131)=103.784, \mathrm{p}<.001]$. Los estudiantes que asisten a establecimientos es- colares de extracción social alta $(\mathrm{M}=22.20, \mathrm{DT}=$ 4.033) evidencian un rendimiento superior al que obtienen los estudiantes de extracción social media $(\mathrm{M}=19.74, \mathrm{DT}=5.025)$ y estos a su vez evidencian un rendimiento superior al de los estudiantes que asisten a establecimientos de extracción social baja $(\mathrm{M}=17.55, \mathrm{DT}=5.337)$.

Al comparar el nivel de inteligencia lógica de los estudiantes del grupo normal de la muestra de investigación con aquellos estudiantes pertenecientes al denominado grupo de estudiantes talentosos, se verifica la existencia de diferencias significativas a favor del grupo de estudiantes talentosos: $(\mathrm{M}=$ $22.32, \mathrm{DT}=4.041)$ y $(\mathrm{M}=18.81, \mathrm{DT}=5.285)$, con lo cual se obtiene $\mathrm{t}(3557)=-12.738, \mathrm{p}<0.001$.

A diferencia de lo que ocurre con la muestra total de estudiantes, en el grupo de estudiantes talentosos el análisis por grupos de extracción social arroja que no existen diferencias significativas entre las puntuaciones medias alcanzadas por cada uno de los grupos en comparación $[\mathrm{F}(2.391)=$ 3.566 , n.s.]. Los estudiantes de grupos sociales altos presentan una media de puntuaciones $(\mathrm{M}=$ 22.85, $\mathrm{DT}=3.764$ ) levemente superior a la de los estudiantes del grupo de extracción social media $(\mathrm{M}=22.25, \mathrm{DT}=4.096)$ y a los que pertenecen a la clase social baja $(\mathrm{M}=21.12, \mathrm{DT}=4.357)$, pero dichas diferencias no resultan ser significativas.

Con respecto a los puntuaciones alcanzadas por hombres y mujeres se observa que los hombres alcanzan un puntaje medio levemente mayor que el de las mujeres $(\mathrm{M}=22.58, \mathrm{DT}=4.036)$ y $(\mathrm{M}=21.98$, $\mathrm{DT}=4.034)$ respectivamente, aunque dichas diferencias tampoco resultan ser estadísticamente significativas $(\mathrm{t}(392)=1.457$, n.s. $)$. 


\section{Discusión}

El IQ Test presenta una distribución que se ajusta a la curva normal con leve asimetría negativa. Desde el punto de vista psicométrico, el IQTest en su versión ajustada es un instrumento válido y fiable. Al sumar esto a su característica de libre acceso a través de internet, se constituye entonces como una herramienta valiosa para examinar la inteligencia lógica de carácter inductiva de la población de estudiantes de educación secundaria chilena. Además, los resultados comparativos permiten establecer categorías que permiten su uso y capacidad de diagnóstico o análisis de impacto diferenciado, dado que permiten establecer una comparación en función del grupo de extracción social y género, evitando sobre o subestimaciones de dichos desempeños. El análisis de los ítems del IQ Test mediante el modelo de Rasch, explicitado en la Figura 2, permite señalar que los ítems finales de la versión depurada se ajustan adecuadamente. Adicionalmente, dado que los parámetros de ítems y personas se expresan en una misma escala (escala logit), la distribución de las medidas de dificultad de los ítems permiten que el test se divida en dos partes equivalentes, lo que favorece su utilización en diseños experimentales de tipo antes-después.

Otro aspecto destacado es su distribución en la dificultad de los ítems, ya que oscila entre -3 y 2 logit en proporciones tales que puede utilizarse de forma masiva en la población objetivo, en este caso, estudiantes de educación secundaria. Al poseer ítems de dificultad bajo la media, estudiantes normales pueden resolver los problemas sin inconvenientes. Por otra parte, también se observa una alta concentración de ítems con dificultad entre 1 y 2 logit, lo que permite discriminar adecuadamente a estudiantes de inteligencia lógica inductiva destacada.

Se comprobó la existencia de diferencias significativas entre las puntuaciones medias alcanzadas por los grupos de estudiantes en función de su edad y nivel de escolaridad. Se observa un incremento consistente a medida que los estudiantes maduran o ascienden en su itinerario educativo. Este resultado es consistente con otras investigaciones relacionadas que prueban que a mayor edad, mayor inteligencia lógico-matemática o matemática, y que estas presentan relaciones positivas, estadísticamente significativas y de magnitud moderada con las sub escalas valoradas en la prueba psicométrica referidas al razonamiento numérico, lógico y nivel cognitivo general (Ferrándiz et al., 2008; Kaufman et al., 2009; Roselli et al., 2009).

Por otro lado, existe una correlación significativa de carácter directamente proporcional entre el desempeño observado en el test y otras áreas de desempeño académico general y en matemáticas. Es decir que los alumnos que presentan buenos desempeños académicos generales y en matemáticas tienden a obtener mejores puntuaciones en el test de inteligencia general y de razonamiento lógico. Esto coincide con el rol destacado que presenta la inteligencia fluida a la hora de explicar la variabilidad del rendimiento escolar, dentro de muchas otras variables, tales como aspectos nutricionales, socio-económicos, familiares y demográficos en el contexto escolar chileno (Ivanovic et al., 2004). El puntaje medio de inteligencia lógica de los hombres es levemente superior que el de las mujeres, no obstante dichas diferencias no resultan ser significativas estadísticamente. Estos resultados concuerdan con los reportados por otras investigaciones (ElseQuest et al., 2010; Ferrándiz et al., 2008; Kaufman et al., 2009; Klein et al, 2010).

También se corroboró que los estudiantes denominados 'talentosos' presentan niveles de inteligencia lógica significativamente superiores a los de estudiantes considerados 'normales'. Los 'estudiantes talentosos' son alumnos que asisten voluntariamente fuera de su jornada escolar habitual a cursos de enriquecimiento matemático o participan de competencias matemáticas,en las que resuelven problemas de un nivel de complejidad superior a los que realizan normalmente en sus clases. Las diferencias encontradas respaldan estudios que sostienen que estos alumnos poseen habilidades cognitivas de alto nivel, tales como la capacidad de planificación, revisión, control, selección y evaluación de sus propias actividades intelectuales, todas ellas de carácter metacognitivo (Onrubia et al., 2003; Sternberg, 2003). 
Más aún, los resultados encontrados en esta investigación son congruentes con otro estudio previo relacionado con alumnos talentosos en Chile que establecía que por sobre las características académicas y socio afectivas-que resultan bastante similares a las de los otros estudiantes-, los alumnos talentosos presentan un mejor dominio específico de destrezas matemáticas, de abstracción y lógica, y geométricas, frente al otro grupo (Flanagan \& Arancibia, 2005). Estos hallazgos difieren de los reportados por un estudio con jóvenes colombianos en el que no se reportaron diferencias significativas entre los grupos de estudiantes talentosos y aquellos de promedio normal. Es decir que de acuerdo al estudio, no existirían diferencias en los niveles de organización, planificación y monitoreo de carácter viso-espacial examinado mediante la prueba de la torre de Hanoi (Montoya, Trujillo, \& Pineda, 2010).

El estudio aporta antecedentes para revalorizar el rol de las denominadas variables cognitivas superiores respecto a los contenidos a la hora de explicar o mejorar el desempeño en el área de matemática o del desempeño académico general. En efecto, aquellos estudiantes que poseen niveles de inteligencia lógica tienden a mostrar desempeños positivos en esta área curricular, independientemente de su edad, sexo y extracción social. Sin embargo, hemos de señalar entre las limitaciones de este estudio, el no haber contado con un análisis más detallado de los contenidos curriculares matemáticos ni de las distintas formas en que estos afectan la práctica de la enseñanza que se imparte, tanto a los escolares de resultados académicos ordinarios como a los que hemos considerado talentosos. Actualmente, lo anterior está siendo sujeto a nuevas investigaciones.

\section{Referencias}

Albright, J., \& Park, H. (2009). Confirmatory Factor Analysis Using Amos, LISREL, Mplus, and SAS/ STAT CALIS. Working Paper. The University Information Technology Services (UITS) Center for Statistical and Mathematical Computing, Indiana University. Recuperado de http://www.indiana. edu/ statmath/stat/all/cfa/index.html
Almeida, L., Guisande, A., Primi, R., \& Lemos, G. (2008). Contributions of the general and the specific factors for the intelligence and school achievement. Relationship. European Journal of Education and Psychology, 1(3), 5-16.

Arden, R., Gottfredson, L., \& Miller, G. (2009). Does a fitness factor contribute to the association between intelligence and health outcomes? Evidence from abnormality counts $3.654 \mathrm{US}$ veterans. Intelligence, 37(6), 581-591.

Blackwell, L., Trzesniewski, K., \& Sorich, C. (2007). Implicit Theories of Intelligence Predict Achievement Across an Adolescent Transition: A Longitudinal Study and an Intervention. Child Development, 78(1), 246-263.

Cerda, G., Ortega, R., Pérez, C., Flores, C., \& Melipillán, R. (2011). Inteligencia lógica y extracción social en estudiantes talentosos y normales de Enseñanza Básica y Media en Chile. Revista Anales de Psicología, 27(2), 389-398.

Cerda, G., Pérez, C., \& Melipillán, R. (2010). Test de Inteligencia Lógica Superior (TILS). Manual de Aplicación. Concepción: Universidad de Concepción.

Cox, C. (2007). Educación en el Bicentenario: dos agendas y calidad de la política. Pensamiento Educativo, 40(1), 175-204.

Crosnoe, R., \& Huston, A. (2007). Socioeconomic Status, Schooling, and the Developmental Trajectories of Adolescents. Developmental Psychology, 43(5), 1097-1110.

Deary, I. J., Strand, S., Smith, P., \& Fernandes, C. (2007). Intelligence and educational achievement. Intelligence, 35(1), 13-21.

Deary, I., Taylor, M. D., Hart, C., Wilson, V., Smith, G., \& Blane, D (2005). Intergenerational social mobility and mid-life status attainment: Influences of childhood intelligence, childhood social factors, and education. Intelligence, 33, 455-472.

Dodonova, Y. A., \& Dodonov, Y. S. (2012). Processing speed and intelligence as predictors of school achievement: Mediation or unique contribution? Intelligence, 40(2), 163-171. doi: http://dx.doi. org/10.1016/j.intell.2012.01.003

Else-Quest, N., Hyde, J., \& Linn, M. (2010). CrossNational Patterns of Gender Differences in Math- 
ematics: A Meta-Analysis. Psychological Bulletin, 136(1), 103-127.

Embretson, S. E., \& Reise, S. (2000). Item response theory for psychologists. Mahwah, NJ: Erlbaum Publishers.

Ferrándiz, C., Bermejo, R., Sainz, M., Ferrando, M., \& Prieto, M. (2008). Estudio del Razonamiento Lógico-Matemático desde el Modelo de las Inteligencias Múltiples. Anales de Psicología , 24(2), 213-222.

Ferreira, A., Almeida, L., Prieto, G., \& Guisande, M. A. (2012). Memória e inteligencia: interdependência em función de los procesos y contenidos de las tareas. Universitas Psychologica, 11(2), 455-467.

Flanagan A., \& Arancibia, V. (2005). Talento Académico: Un Análisis de la Identificación de Alumnos Talentosos Efectuada por Profesores. Psykhe, 14(1), 121-135.

Furnham, A., Monsen, J., \& Ahmetoglu, G. (2009). Typical intellectual engagement, big five personality traits, approaches to learning and cognitive ability predictors of academic performance. British Journal of Educational Psychology, 79(4), 769-782.

García-Cepero, M., \& McCoach, D.B. (2009). Educators' implicit theories of intelligence and beliefs about the identification of gifted students. Universitas Psychologica, 8(2), 295-310.

García-Huidobro, J. E., \& Bellei, C. (2003). Desigualdad educativa en Chile. Santiago: Universidad Alberto Hurtado.

Gottfredson, L., \& Deary, I. (2004). Intelligence predicts health and longevity, but why?. Current Directions in Psychological Science, 13, 1-4.

Gullick, M. M., Sprute, L. A., \& Temple, E. (2011). Individual differences in working memory, nonverbal IQ, and mathematics achievement and brain mechanisms associated with symbolic and nonsymbolic number processing. Learning and Individual Differences, 21, 644-654. doi: http://dx.doi. org/10.1016/j.lindif.2010.10.003

Ivanovic, D., Pérez, H., Olivares, M., Díaz, N., Leyton, B., \& Ivanovic, R. (2004). Scholastic Achievement: A Multivariate Analysis of Nutritional, Intellectual, Socioeconomic, Sociocultural, Familial, and Demographic Variables in Chilean School-Age Children. Nutrition, 20(10), 877-889.
Johnson, W., \& Bouchard, J. (2005). The Structure of Human Intelligence: Perceptual, and Image Rotation (VPR), Not Fluid and Crystallized. Intelligence, 33(4), 393-416.

Kaufman, A., Kaufman, J., Liu, X., \& Johnson, C. (2009). How do educational attainment and gender relate to Gf, Gc, and academic skills at ages 22-90 years? Archives of Clinical Neuropsychology. doi: http://dx.doi.org/10.1016/j.acn.2008.12.001.

Klein, P., Adi-Japha, E., \& Hakak-Benizri, S. (2010). Mathematical thinking of kindergarten boys and girls: similar achievement, different contributing processes. Educational Studies in Mathematics, 73, 233-246.

Kornilova, T., Kornilov, S., \& Chumakova, M. (2009). Subjective evaluations of intelligence and academic self-concept predict academic achievement: Evidence from a selective student population. Learning and Individual Differences, 19, 596-608.

Kotz, K., Watkins, M., \& McDermott, P. (2010). Validity of the general conceptual ability score from the differential Ability Scales as a function of significant and rare interfactor variability. School Psychology Review, 37, 261-278.

Kvist, A. V., \& Gustafsson, J. (2008). The relation between fluid intelligence and the general factor as a function of cultural background: a test of Catell's investment theory. Intelligence 36, 422-436.

Linacre, J. M. (2010). Winsteps (Version 3.70.02) [Computer Software]. Chicago:Winsteps.com.

Lynn, R., \& Mikk, J. (2009). National IQs predict educational attainment in math, reading and science across 56 nations. Intelligence, 37, 305-310.

MINEDUC (2007a). PISA 2006: Rendimientos de estudiantes de 15 años en Ciencias, Lectura y Matemática. Chile: Ministerio de Educación, Unidad de Currículum y Evaluación.

MINEDUC (2007b). Niveles de Logro 4o Básico Lectura y Educación Matemática SIMCE. Chile: Ministerio de Educación, Unidad de Curriculum y Evaluación.

MINEDUC (2010a). Resultados Nacionales SIMCE. Chile: Ministerio de Educación, Unidad de Curriculum y Evaluación. 
MINEDUC (2010b). Resumen de Resultados PISA 2009 Chile. Chile: Ministerio de Educación, Unidad de Curriculum y Evaluación.

Montoya, D., Trujillo, N., \& Pineda, D. (2010). Capacidad intelectual y función ejecutiva en niños intelectualmente talentosos y en niños con inteligencia promedio. Universitas Psychologica, 9(3), 737-747.

Muñiz, J. (2010). Las Teorías de los tests: teoría clásica y teoría de respuesta a los ítems. Papeles del Psicólogo, 31(1), 57-66.

Onrubia, J., Rochera, M., \& Barberá, E. (2003). La Enseñanza y el Aprendizaje de las Matemáticas: Una Perspectiva Psicológica. En Palacios, J., Marchesi, A., \& Coll, C. (Eds.). Desarrollo Psicológico y Educación 1. Psicología evolutiva (pp. 453-469). Madrid: Alianza.

Raven, J., Raven, J. C., \& Court, J. H. (1993). Test de Matrices Progresivas. Escalas Coloreada, General y Avanzada. Buenos Aires: Paidos.

Rindermann, H. (2008). Relevance of education and intelligence for the political development of na- tions: Democracy, rule of law and political liberty. Intelligence, 36, 306-322.

Roselli, M., Ardila, A., Matute, E., \& Inozemtseva, O. (2009). Differences and Cognitive Correlates of Mathematical Skills in School-Aged Children. Child Neuropsychology, 15(3), 216-231.

Spinath, B., Freunderthaler, H., \& Neubauer, A. (2010). Domain-specific school achievement in boys and girls as predicted by intelligence, personality and motivation. Personality and Individual Differences, 48, 481-486.

Steinmayr, R., Ziegler, M., \& Träuble, B. (2010). Do intelligence and sustained attention interact in predicting academic achievement? Learning and Individual Differences, 20, 14-18.

Sternberg, R. (2003). What is an "Expert Student". Educational Researcher, 32(8), 5-9.

Watkins, M., Lei, P-W., \& Canivez, G. (2007). Psychometric intelligence and achievement. A crosslagged panel analysis. Intelligence, 35, 59-68. doi: http://dx.doi.org/10.1016/j.intell.2006.04.005 